\title{
Integrative Medicine, the Direction of Future Medicine: An Interview with Prof. Wenjian Wang
}

\author{
Weidong Pan \\ Department of Neurology, Shuguang Hospital Affiliated to Shanghai University of \\ Traditional Chinese Medicine, Shanghai, China
}

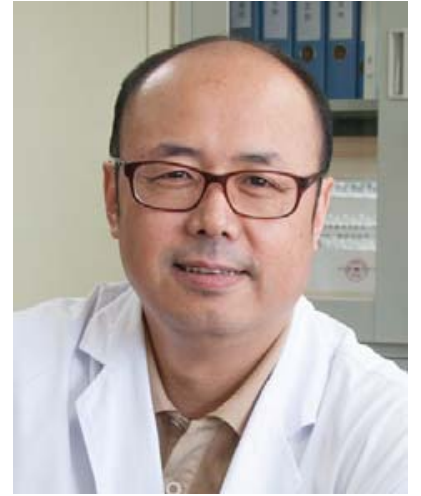

Weidong Pan, MD, PhD.

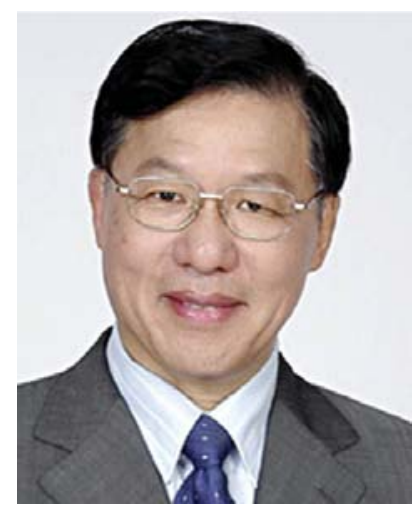

Prof. Wenjian Wang.

\section{Curriculum Vitae}

Prof. Wenjian Wang is currently a well-known Traditional Chinese Medicine (TCM) doctor and doctoral tutor of Fudan University. Leadership positions held by Prof. Wang include the presidency of the Integrative Medicine Institute and College of Integrative Medicine of Fudan University. He is also the vice chairman of the China Association of Integrated Traditional and Western Medicine, the president of the Shanghai Association of Chinese Integrative Medicine, and director of the major discipline of Integrative Medicine for the Chinese Ministry of Education. He is editor or associate editor of many journals, such as the Chinese Journal of Integrated Traditional and Western Medicine, Journal of Chinese Integrative Medicine, Chinese Patent Medicine, and Journal for Difficult Diseases, and serves as an editorial board member of many medical journals published in China and other countries. Prof. Wang is an internationally recognized integrative medicine expert of endocrine and metabolic diseases as well as cardiovascular and cerebral vascular diseases. In the late 2000s, 
he established a highly successful program focusing on the development of integrative medicine. More recently, he has published more than 100 articles in renowned international periodicals that focus mainly on integrative medicine, and he has received science and technology progress awards from the Traditional Chinese Medicine Administration Bureau and from The People's Government of Shanghai on 7 occasions.

\section{Q: What Do You Think about the Connections between the Ancient Systems of TCM and Western Medicine?}

The initial medical systems of both TCM and Western medicine were developed more than 2,000 years ago, although TCM had a more integrated and advanced system in the initial stages than Western medicine. Medical text books by Huang Di Nei Jing (210 BCE), Shang Han Za Bing Lun (300 CE), and Qian Jin Yao Fang (652 CE) established the initial TCM system and guided TCM doctors in the treatment of most diseases. There were many famous doctors such as Bian Que (407-310 BCE), Hua Tuo (145-208 CE), and Zhang Zhongjing (150-215 CE). Doctors of Chinese medicine could treat most diseases including cholera, influenza, dysentery, and cancer and could even perform brain surgery using herbal anesthetics (Ma Fei San by Hua Tuo). Physical treatments such as acupuncture were also well developed. Acupuncture was first described by Huang Di Nei Jing and developed in Zhen Jiu Jia Yi Jing (259 CE) by Huang Pumi (215-282 CE). The ancient medical system of TCM had a very advanced level for treating many diseases, and the theory included most systems of the body. The medical system was not divided into independent systems because the doctors thought of the human body as one integrated system. Most people believe that Western medicine was started by Hippocrates (460-377 BCE) and developed from Galen (129-199 CE). Hippocrates separated the discipline of medicine from religion, believing and arguing that disease was not a punishment inflicted by the gods but rather the product of environmental factors, diet, and living habits. He started treating diseases with medicines that included some traditional materials from plants such as olive oil. He is referred to as the 'Father of Western medicine'. Galen's understanding of anatomy and medicine was principally influenced by the current theory of humorism, which involves dividing the human body into systematic recognition. His theories dominated and influenced Western medical science for more than 1,300 years. According to the history of the two medical systems, they have their own scientific basis and specificity.

\section{Q: What Do You Think about the Necessity to Establish Interdisciplinary Associations in Modern Society?}

We should establish interdisciplinary associations to study and treat interdisciplinary diseases. In the ancient medicine systems, not only Western medicine but also TCM, doctors considered diseases through an integrated view (consideration of the whole body). With the development of clinical medicine, biomedicine, and scientific technology, especially in the Western medicine system, doctors started to divide the human body into many independent systems for more advanced diagnoses, clinical management, and clinical research. In modern society, we can find more and more single-system clinical experts and scientists who conduct high-quality specialized and experimental research and treatment of various diseases. These scientists and doctors, of course, are necessary and beneficial for treating these diseases, but they might neglect or overlook something. The human body is not only an object but also a living creature. All of the systems in our body, such as the nervous, circulatory, digestive, endocrine, urinary, and reproductive systems, do not exist in isolation but rather are inte- 


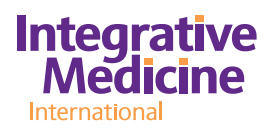

\begin{tabular}{l|l}
\hline Integr Med Int 2015;2:139-142 \\
\hline DOI: 10.1159/000441123 & $\begin{array}{l}\text { (c) 2015 The Author(s). Published by S. Karger AG, Basel } \\
\text { www.karger.com/imi }\end{array}$ \\
\hline
\end{tabular}

Pan: Integrative Medicine, the Direction of Future Medicine: An Interview with Prof. Wenjian Wang

grated as one whole object. For example, when a person suffers from prostatic cancer, he should be treated by endocrine therapy. Some neurological diseases, such as Parkinson's disease, Alzheimer's disease, epilepsy, and encephalitis, are closely related to the endocrine system. In modern society, we need interdisciplinary doctors to integrate medicine to deal with these diseases in the way ancient doctors did.

\section{Q: What Do You Think about the Treatment of Cancer in Modern Society?}

The occurrence and development of cancer are very complicated and might be related to disorders of immunity and of the endocrine system and are not a single-organ problem. Based on the theory of Western medicine, we have to determine which organ or tissue is affected by the cancer. The principal guideline of Western medicine when treating cancer is: 'early detection, early treatment', and 'special cancer, special treatment'. They use surgery, chemotherapy, radiotherapy, and targeted therapy to deal with the cancer. The treatments sometimes work very well for the cancer; however, they may be accompanied by many side effects and even contribute to the death of the patient. The morbidity of a cancer may be caused by the chemotherapy or radiotherapy of Western medicine through the destruction of the integrated endocrine balance and immunity of the body. In TCM theory, the body has a reserve ability to resist any harmful factors which are called 'Xie Qi' (cancer cells), and the reserve ability is called 'Zheng Qi'. Zheng Qi can balance the internal environment to resist or destroy the growth of Xie Qi (cancer cells). We call this phenomenon 'Zheng Xie Xiang Zheng' (the struggle between Zheng Qi and Xie Qi). If the Xie Qi prevails over the Zheng Qi, it might cause cancer or other problems. If the Zheng Qi is more powerful than the Xie Qi, the body will ultimately recover. According to theory, if cancer occurs, TCM doctors should treat the cancer in two ways. One is to adjust the balance of the internal environment in order to increase the power of Zheng Qi by the 'Bian Zheng Shi Zhi' method. The other way is to decrease the Xie Qi (cancer cells) by aggressive therapies. TCM doctors have had more success than Western medicine in treating liver cancer, prostatic carcinoma, leukemia, and others using these methods, although many unsuccessful cases are still encountered. TCM therapy expresses a more relevant meaning of 'integrative medicine' for cancer than Western medicine.

\section{Q: Do You Agree that TCM Is Better at Treating Chronic Diseases than Western Medicine?}

TCM is not only effective for chronic diseases but also for acute and epidemic diseases. There is a lot of evidence indicating that TCM can modulate many unbalances in the human body, and TCM has been used for many chronic diseases. Approximately 2,000 years ago, the era of TCM developed rapidly due to a series of plagues which led to the death of $70 \%$ of the family members of Dr. Zhongjing Zhang. Through protracted and unremitting efforts, Dr. Zhang cured many patients seriously affected by the plagues, and he ultimately became a famous doctor and published one monumental text book, Shang Han Za Bing Lun, which instructed doctors on how to treat most acute and chronic diseases known at that time. We all must remember the terrible outbreak of severe acute respiratory syndrome (SARS) about 10 years ago. TCM supplied effective component medicine in the treatment of SARS, and it was reported that China had less morbidity in SARS patients, especially in those treated at TCM hospitals, than patients in Western countries. TCM exhibits much stronger effects than Western medicine in the treatment of acute diseases. TCM has also shown potent efficacy against acute appendicitis, acute hepatitis, and acute pneumonia. 


\section{Integrative Medicine}

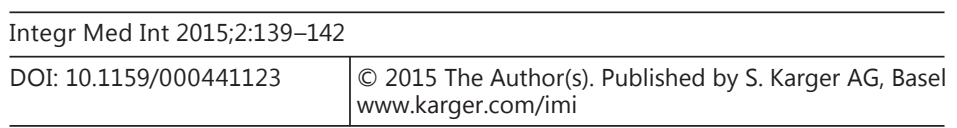

Pan: Integrative Medicine, the Direction of Future Medicine: An Interview with Prof. Wenjian Wang

\section{Q: What Do You Think about the Scientific Integrity and Robustness of TCM?}

Of course, TCM has scientific integrity and is robust. TCM comes from clinical practice throughout a long history in China, and many Chinese doctors observe the symptoms of patients using simple natural treatments based on traditional Chinese dialectical theory. Through the development of TCM, they eliminated many inappropriate methods and materials and increased useful treatments by the development of science and technology in China. Even in the late 1840s, by the importation of Western medicine and modern technology, modern TCM doctors corrected many of the weaknesses of TCM by studying and applying new biomedical and pathological principles obtained from Western medicine. TCM has its own rigor theory and treatment system and treats diseases accurately step by step with less side effects than Western medicine.

\section{Q: What Is Health? What Is the Difference between the Concept of Health in TCM and Western Medicine?}

Health is not merely the 'absence' of pathology. The concept of health should be based on physical and mental health. It is not only anoesis of the body but also anoesis of emotion, mind, and feeling. TCM emphasizes that integrative medicine is more humanistic and reasonable for human health than Western medicine. Most modern Western doctors believe, based on the guidelines of Western medicine, that if a person has high blood pressure with no uncomfortable symptoms, he or she should take medicine to control the blood pressure to prevent cardiovascular and/or brain vascular diseases. If no symptoms are present and only hyperlipidemia is revealed in laboratory tests, is the person healthy? The person is not a patient but still has to take hypolipidemic drugs. Human beings do not exist in a biological vacuum. We live in interdependent existence with the totality of the living world [1]. In TCM theory, these people might be healthy because they do not have any incompatibility in their life. Western physicians might pay more attention to the laboratory indicators and physical tests, while TCM doctors might pay more attention to the feelings of the individual concerning personality, activities of daily living, quality of life, and appetite. Neither theory encompasses the whole meaning of health. I agreed with the concept that 'positive' and 'health' are synonyms [2]. Positive health is something different from the health of ordinary persons. Mental illness is a condition that can be defined reliably compared with positive mental health. The interactions between physical, psychological, and social health remain powerfully relevant to this day [3]. Human health cannot be separated from the health of our total planetary biodiversity. The integrated meaning of Western medicine and TCM is the modern concept of health, which means 'state of complete physical, mental, and social well-being'. According to the modern concept of health, high blood pressure and hyperlipidemia should be controlled, a suitable diet should be consumed, and physical activity such as exercise should be undertaken, even if the person does not feel any discomfort, not only to return to normal laboratory test levels but also to produce the preventive ability (Zheng Qi) to increase positive health. This is the concept of modern health.

\section{References}

\footnotetext{
Linden M: What is health and what is positive? The ICF solution. World Psychiatry 2012;11:104-105. What is health? The ability to adapt. Lancet 2009;373:781.

Pan W, Zhou H: Inclusion of integrative medicine in clinical practice. Integr Med Int 2014;1:1-4.
} 